\title{
A system preventing children from getting hit while passing by the front based on laser sensor
}

\author{
Chen Xiaowei ${ }^{1, a}, N_{i u} W_{e n y u^{2, b}}$, Luo Yinping ${ }^{3 C}$, Yang Jie ${ }^{4, d}$, Chen Xiaowei ${ }^{5, e}$ \\ ${ }^{1}$ WuhanUniversity of Technology, Wuhan 430000, China; \\ ${ }^{2}$ WuhanUniversity of Technology, Wuhan 430000, China.

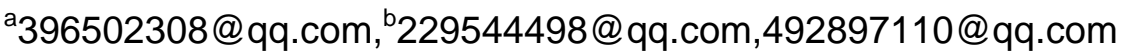

\section{Keywords: 51 SCM; Laser sensor; Radar}

\begin{abstract}
A system preventing children from getting hit while passing by the front based on laser sensor aims at avoiding knocking down the child while passing by the front after getting off the bus, owing to the blind spot of the front to the driver. The system mainly consists of SCM, laser sensors and radar sensors. It is the two laser sensors installed inside and outside that minimize the child casualties by starting detection of the reverse radar sensors opportunely.
\end{abstract}

\section{Introduction}

The blind area of drivers gives rise to accidents affecting the safety of children who walk around the front of the school bus after getting off. At present, the methods, for example, adding front mirrors and telescopic manipulator, are applied currently in the foreign countries. But it's still difficult to solve the problem fundamentally. Therefore, how to alarm the driver accurately and immediately plays a key role in these accidents.

\section{The overall design of the system}

The system is made up of three parts - starting unit, controlling unit, detecting and response unit. The design detail follows. Starting unit is composed of the two laser sensors mounted closely on the door of the school bus, in order to judge when the children get off. Controlling unit including 51 SCM and switch relay is conducive to receive the signal of laser sensors and dominate radar mainframe box. Detecting and response unit is formed by two components, radar probe and main engine box, installed on the front of the school bus to test the passing child and respond immediately.

Workflow of the system is as follows. When a child gets on or off, the two laser sensors will obtain signal in a different anterior-posterior. If the laser sensor closed to outside detects the signal firstly and the inside laser sensor detects the signal secondly, the system will know that the child is getting on and the SCM needn't any controlling operation. On the contrary, if the inside laser sensors detect the signal firstly and the outside laser sensor detect the signal secondly, the system will know that the child is getting off, which urges the SCM to start the relay and the radar mainframe box begin to work. On the basis of the second situation that has detected the child is passing by the front of the school bus, the radar probe will get the ultrasonic wave back, the buzzer in host box will work immediately to warn of the driver. The workflow is shown in Fig. 1.1. 


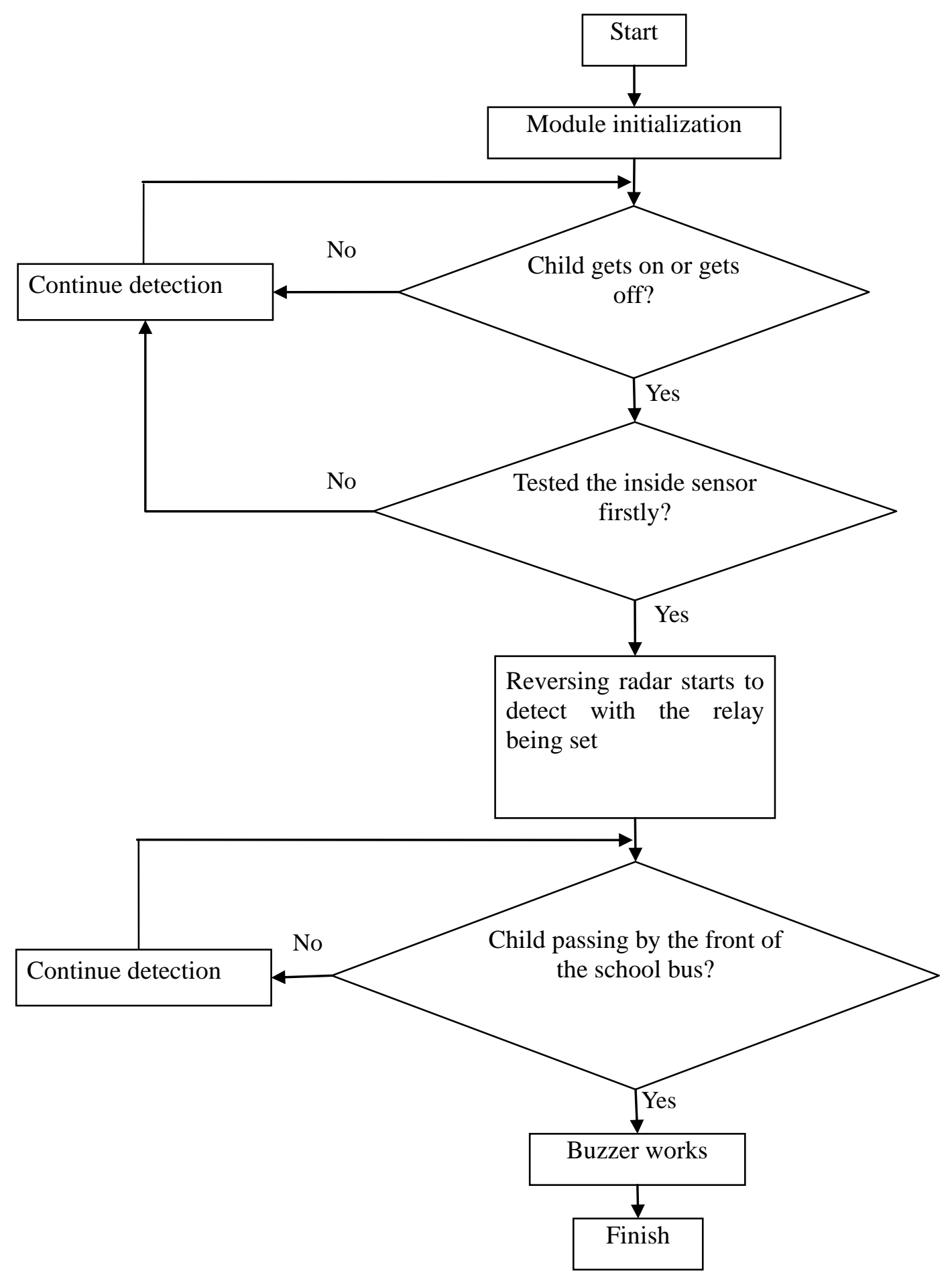

Fig. 1.1 the workflow of the system

\section{Hardware Design}

\subsection{Each hardware module's parameter selection}

The system's control unit is STC89C52 microcontroller, whose program memory space is 8K bytes, data storage space is 512 bytes, power provided is $5 \mathrm{~V}$ supply, and $\mathrm{P} 1$ is the quasi-bidirectional/weak pull, therefore it does not need to add an external pull-up resistor to do the function as I/O port. The radar sensor uses dual-core LED 4 probe buzzer, which ensures its testing distance between $0.4 \mathrm{~m}$ and $2.0 \mathrm{~m}$, and its alarm volume over $80 \mathrm{~dB}$. The laser sensor adopt M12 cylindrical correlative laser photoelectric switch, and its function parameters are shown in Table 1.1 . 
Table 1.1 Laser photoelectric switch function parameters

\begin{tabular}{|c|c|}
\hline Electric current & $200 \mathrm{~mA}$ \\
\hline Voltage & DC5V \\
\hline Testing distance & $1 \sim 8 \mathrm{~m}$ \\
\hline Outer material & Nickel -plated brass \\
\hline Degrees of protection & TP66 \\
\hline Response time & Correlation type: $<1 \mathrm{~ms}$ \\
\hline Anti-interference capability & Remain undisturbed in daylight \\
\hline Functional advantage & Small laser emission angle and several kinds of beam \\
\hline Ambient light illumination & daylight lamp: $\leq 3000 \mathrm{Lx} /$ daylight: $\leq 10000 \mathrm{Lx}$ \\
\hline
\end{tabular}

\subsection{Overall circuit design system}

MCU clock circuit uses HC49/S-HC/49SS passive crystal, which connects interface XTAL1 and XTAL2 with other two 30pF ceramic capacitors and its nominal frequency is $11.0592 \mathrm{MHZ}$; Reset circuit using buttons and electrolytic capacitors series connection; The two laser sensors are connected with each other respectively in the external microcontroller interrupt port INT0 and INT1. When the laser sensor with light passing through, the receiving tube OUT port is of high level, and conversely, it is low level of shading. Simultaneously, the internal sensor is connected with the external interruption 0 , and the external sensor is connected with the external interruption 1 . The relay control terminal is connected to P2.0 port of SCM. When the P2.0 level is high, the relay starts to work, the normally open contact is closed, and the normally closed contact is disconnected.

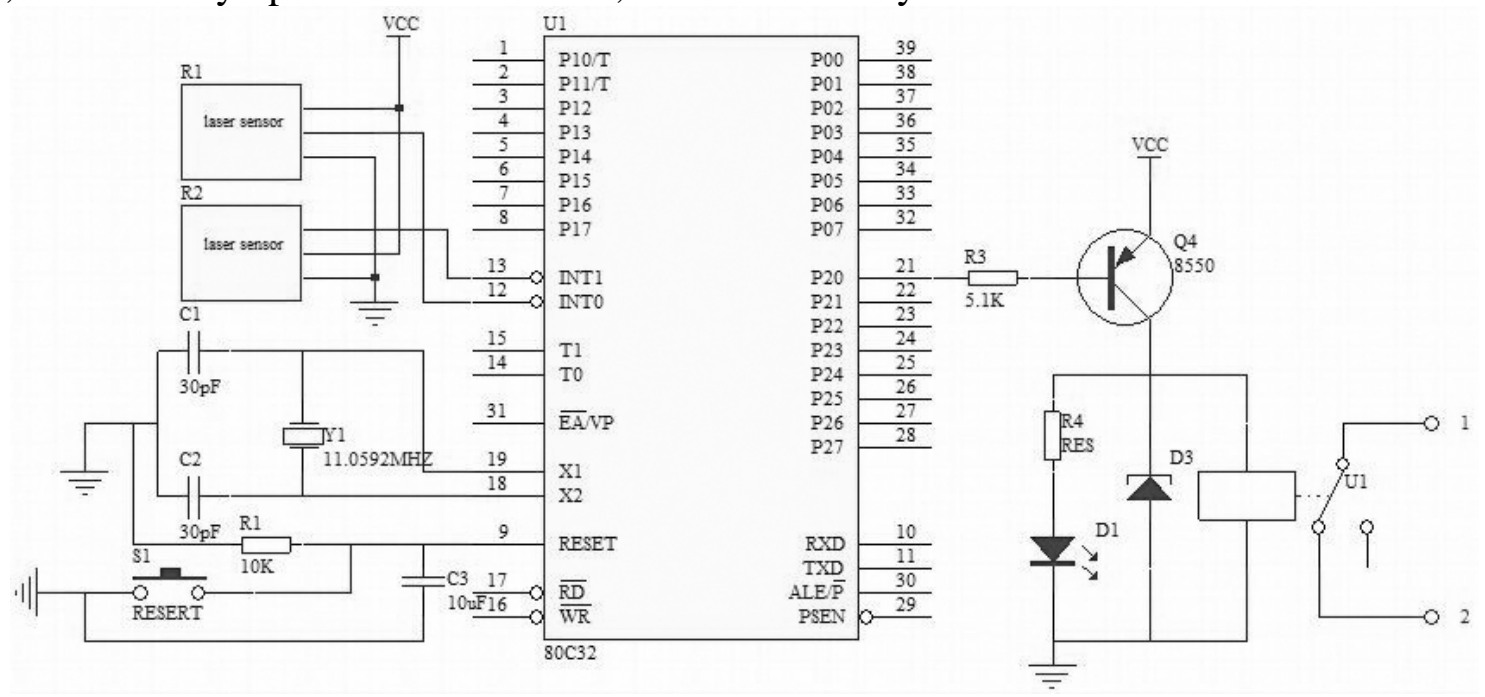

Fig.1.3 Overall system schematic

\section{Software Design}

System program design consists of detecting module, judgment module and controlling module. A pair of laser sensors is composed of transmitting tubes and receiving tubes. If the transmitting tubes send out laser signal to the receiving tubes without any obstacles, the latter will output high power level. However, once the laser path is blocked, the output port will transform from high level to low level. Therefore, in the circumstance that they are connected to the external microcontroller 
interrupt port, we can judge whether there is something passing by with setting the effective mode as low level. The algorithm of detecting the child is getting on is entering the external interruption 1 firstly and the external interruption 0 later. On the contrary, the algorithm of detecting the child is getting off is entering the external interruption 0firstly and the external interruption 1 later. The algorithm design is shown in Fig. 1.4. After judging the child has got off, the system shall start the relay and the radar mainframe box, the radar probe begins to detect, and the buzzer in the main engine box alarms automatically as long as there is a child passing by.

Mounted closed to inside
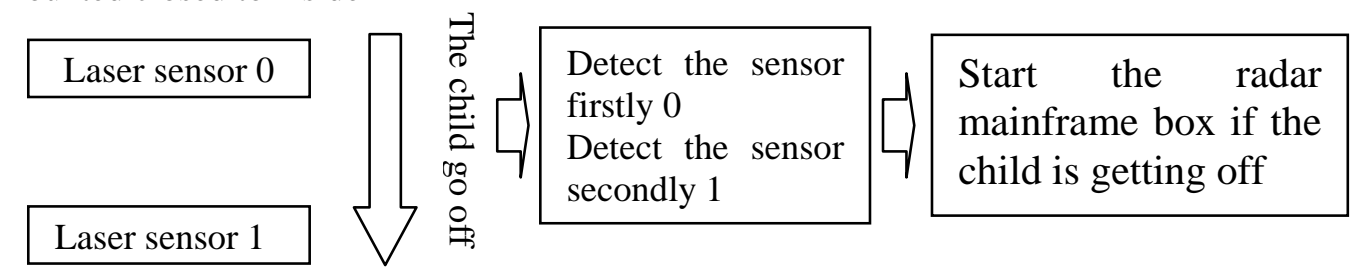

Mounted closed to outside

Fig. 1.4 Algorithm design

\section{Prospects and Conclusions}

A system preventing children from getting hit while passing by the front based on laser sensor aims at avoiding knocking down the child while passing by the front after getting off the bus, owing to the blind spot of the front to the driver. It is the two laser sensors installed inside and outside that minimize the child casualties by starting detection of the reverse radar sensors opportunely.It has broad application prospects and good feasibility.

\section{References}

[1] Min Woo Park,Kyung Ho Jang,Soon Ki Jung. Panoramic Vision System to Eliminate Driver’ s Blind Spots using a Laser Sensor and Cameras[J]. International Journal of Intelligent Transportation Systems Research,2012,103:3-15.

[2] Hiroki Sakai,Tomoo Shiigi,Naoshi Kondo,Yuichi Ogawa,Nobuyoshi Taguchi. Accurate Position Detecting during Asparagus Spear Harvesting using a Laser Sensor[J]. Engineering in Agriculture, Environment and Food,2013,63:4-23.

[3] O. Méndez-Zepeda,S. Muñoz-Aguirre,G. Beltrán-Pérez,J. Castillo-Mixcóatl. Alternative interrogation method for a dual laser sensor based on fiber Bragg gratings to measure temperature using the fundamental beating frequency intensity[J]. Optics and Laser Technology,2014,:5-13.

[4] Bettez Neil D,Groffman Peter M. Denitrification potential in stormwater control structures and natural riparian zones in an urban landscape.[J]. Environmental Science \&amp; Technology,2012,4620:6-24.

[5] Dmitry Ivanov,Alexandre Dolgui,Boris Sokolov. Applicability of optimal control theory to adaptive supply chain planning and scheduling[J]. Annual Reviews in Control,2012,361:7-16.

[6] Anonymous. SCM Microsystems, Inc.: SCM Microsystems and Bluehill ID Agree to Combine in an All Share Transaction; Combination Aims To Create A New Leader In Access Control, Identity Management And Rfid Technologies[J]. M2 Presswire,2009,:23-34. 